besteht ein linearer Zusammenhang mit $H$, sofern das Magnetfeld in der Richtung [100] oder [111] liegt. Für ein Magnetfeld in Richtung [110] erhält man einen Sättigungswert. Wie FrIE zeigen kann, ergibt sich mit Hilfe seiner Theorie aus den linearen Kurven der Abb. 9 (Probe 15) ein durchaus vernünftiger Wert für die Stoßzahl, so daß eine befriedigende Übereinstimmung besteht.
Abschließend möchten wir Herrn Prof. Justi für die Möglichkeit zur experimentellen Durchführung dieser Arbeit herzlich danken. Besonderer Dank gilt den Herren Prof. Sснотткy und Prof. Franz für wertvolle Hinweise und Diskussionen. Die Einkristalle wurden uns dankenswerterweise von Herrn F. W. Deнmelt (Telefunken) zur Verfügung gestellt. Die Deutsche For $\mathrm{schungsgemeinschaft} \mathrm{hat} \mathrm{die} \mathrm{Untersuchungen}$ durch Sachbeihilfen und Leihgaben großzügig unterstützt.

\title{
Theorie der Kleinwinkelstreuung von Röntgen-Strahlen und Neutronen durch innere Spannungen in festen Körpern, insbesondere durch Versetzungen
}

\author{
Von Alfred Seeger und Ekkehart Kröner \\ Aus dem Institut für theoretische und angewandte Physik der Technischen Hochschule Stuttgart \\ und dem Max-Planck-Institut für Metallforschung, Stuttgart \\ (Z. Naturforschg. 14 a, $74-80$ [1959]; eingegangen am 3. September 1958)
}

\begin{abstract}
Die von Versetzungen herrührende Kleinwinkelstreuung, die bisher als Integral über das Dilatatationsfeld der Versetzungen (und anderer Fehlstellen) ermittelt werden mußte, wird (soweit sie sich nach der linearen Elastizitätstheorie behandeln läßt) als Integral über die Versetzungsdichte dargestellt. Für Versetzungslinien (sog. singuläre Versetzungen) reduziert sich dieses Integral auf ein entlang der Versetzungslinien zu erstreckendes Linienintegral, mit dessen Hilfe die Rechnungen über die Kleinwinkelstreuung von Versetzungen sehr vereinfacht werden. Als Beispiel wird die Kleinwinkelstreuung durch kreisförmige Stufenversetzungsringe behandelt. Solche Ringe können durch die Kondensation von Leerstellen in Kristallen entstehen und spielen neuerdings eine Rolle bei der Diskussion der Vorgänge in abgeschreckten Metallen.
\end{abstract}

Für die Streuung von Röntgen-Strahlen ist die Verteilung der Elektronen maßgebend. Betrachtet man einen Kristall einheitlicher chemischer Zusammensetzung, insbesondere einen Elementkristall (z. B. ein Metall), so ist bei genügend großer Ordnungszahl die Elektronendichte praktisch proportional der Dichte der Materie, da man unter diesen Bedingungen den Einfluß der Valenzelektronen neben demjenigen der Rumpfelektronen vernachlässigen kann. Durch die Kleinwinkelstreuung von RöNTGENStrahlen werden bekanntlich die Variationen in der Elektronenverteilung, die durch die atomare Struktur der Kristalle bedingt sind, nicht erfaßt, wohl aber diejenigen Dichtevariationen, die sich über eine größere Zahl von Atomabständen hinweg erstrekken. Für die Beschreibung solcher Dichtevariationen eignet sich naturgemäß die Elastizitätstheorie besonders gut, so daß man zur Behandlung der Kleinwinkelstreuung durch innere Spannungen in festen Körpern zweckmäßigerweise von der im Sinne der Kontinuumsgeometrie definierten Volumdilatation $\Theta(\mathfrak{r})$ als Funktion des Ortsvektors $\mathfrak{r}$ ausgeht. Man kann zeigen ${ }^{1}$, daß die Amplitude der Kleinwinkel- streuung näherungsweise durch die dreidimensionale Fourier-Transformierte der Dilatation, also durch den Ausdruck

$$
A(\mathfrak{g})=\iiint \Theta(\mathfrak{r}) \exp (-i \mathfrak{g} \cdot \mathfrak{r}) \mathrm{d} \tau_{\mathrm{r}},
$$

erstreckt über den ganzen Kristall, bestimmt ist. Hierbei ist der sog. Beugungsvektor $\mathfrak{g}$ als vektorielle Differenz zwischen dem Wellenvektor $\mathfrak{f}^{\prime}$ der gestreuten und dem Wellenvektor $\mathfrak{f}$ der einfallenden Welle, also durch

$$
\mathfrak{g}=\mathfrak{f}^{\prime}-\mathfrak{f},
$$

definiert. Bezeichnet $2 \vartheta$ den sog. Streuwinkel, d. h. den Winkel zwischen $\mathfrak{f}^{\prime}$ und $\mathfrak{f}$, so hängt der Betrag $g$ des Beugungsvektors mit der Wellenlänge $\lambda$ gemäß

$$
g=\frac{4 \pi \sin \vartheta}{\lambda}
$$

zusammen ${ }^{2}$.

1 Siehe hierzu z. B. A. Guinier u. G. Fournet, Small-Angle Scattering of X-Rays, John Wiley \& Sons, Inc., New York 1955 sowie A. SEeger, International Conference on the Small-Angle Scattering from Metals, Kansas City 1958 (Veröffentl. J. appl. Phys., demnächst).

2 Diese Bezeichnungen sind dieselben, wie sie früher verwendet wurden (A.SEEGER,Z.Naturforschg.11 a,724 [1956]). 
Gl. (1) gilt auch für die Streuung langsamer Neutronen, da deren Streuung an den Kernen erfolgt und damit ebenfalls durch die Dichte der Materie bestimmt ist.

Bei der Anwendung der Gl. (1) auf innere Spannungen in Kristallen ist es zweckmäßig, das Hauptaugenmerk auf die Kleinwinkelstreuung von Versetzungen zu richten, da die Versetzungen die elementaren Quellen der Eigenspannungen darstellen und sich beliebige Eigenspannungsverteilungen durch die Überlagerung von Versetzungen darstellen lassen ${ }^{3}$.

In der Literatur wurde die Kleinwinkelstreuung durch Versetzungen in einer Reihe von speziellen Fällen behandelt ${ }^{4,5,6,7,8,9,10}$. Dabei wurde so vorgegangen, daß mit Hilfe der Elastizitätstheorie die Volumdilatation $\Theta(\mathfrak{r})$ in der Umgebung der Versetzungslinie berechnet und in das FourIer-Integral Gl. (1) eingeführt wurde. Verläuft eine Versetzungslinie jedoch nicht geradlinig, so wird der Ausdruck für das Verschiebungsfeld und für die Dilatation selbst bei Beschränkung auf die lineare Elastizitätstheorie und auf isotrope Medien so kompliziert, daß eine einfache und übersichtliche Auswertung der Gl. (1) für krummlinig verlaufende Versetzungen auf diesem Wege nicht möglich erscheint. Es besteht daher das Bedürfnis nach einem einfachen und allgemein brauchbaren Verfahren zur Berechnung der Kleinwinkelstreuung durch Versetzungen. Eines der Ziele der vorliegenden Arbeit ist es, ein solches Verfahren zu entwickeln. Wir werden in Abschnitt 1 zeigen, daß es, sofern man sich auf den Gültigkeitsbereich der linearen Elastizitätstheorie beschränkt, bei beliebigem Verlauf der Versetzung möglich ist, die Amplitude $A(\mathfrak{g})$ als ein verhältnismäßig einfaches Linienintegral über die Versetzungslinie darzustellen. Die Tragweite der Methode werden wir im Abschnitt 2 dadurch demonstrieren, daß wir ein bisher ungelöstes, aber in neuester Zeit vom experimentellen Standpunkt aus besonders wichtig gewordenes Problem, nämlich die Kleinwinkelstreuung durch einen kreisförmigen Versetzungsring, auf einfache Weise und in geschlossener Form lösen. Diese Rechnungen werden der Einfachheit halber für

3 E. KRöNer, Kontinuumstheorie der Versetzungen und Eigenspannungen. Springer-Verlag, Berlin-Göttingen-Heidelberg 1958.

${ }^{4}$ D. L. Dexter, Phys. Rev. 90, 1007 [1953].

5 J. Burn, Diffusion Centrale des Rayons X par les Métaux (Dissertation Paris 1954), Imp. du Ministère de l'Air, Paris 1956. elastisch isotrope Medien (z. B. Aluminium) durchgeführt. Wir zeigen in einem Anhang am Beispiel eines kubischen Kristalls, daß sich die Methode ohne allzugroßen Mehraufwand auch auf anisotrope Medien erweitern läßt.

\section{Die Kleinwinkelstreuung durch Versetzungs- linien}

Für die Amplitude der Kleinwinkelstreuung durch Versetzungslinien ist, wie Gl. (1) zeigt, die von den Versetzungen hervorgerufene Volumdilatation $\Theta$ maßgebend. $\Theta$ umfaßt sowohl einen durch die lineare Elastizitätstheorie gegebenen wie auch einen nur durch Anwendung der nichtlinearen Elastizitätstheorie oder die Gittertheorie berechenbaren Beitrag. In der vorliegenden Arbeit werden wir uns mit dem erstgenannten Anteil befassen, der sich in mathematisch besonders einfacher Weise behandeln läßt und für viele Fragestellungen, z. B. das in Abschnitt 2 zu behandelnde Beispiel, die Hauptrolle spielt.

Die Berücksichtigung des durch die Anwendung der linearen Elastizitätstheorie nicht erfaßbaren Anteils ist erheblich schwieriger, und zwar schon allein deswegen, weil die hierfür maßgebenden Materialkonstanten nicht in genügendem Umfange bekannt sind. Beim heutigen Stand der Kenntnisse hat man sich hierbei im wesentlichen auf die Anwendung der Elastizitätstheorie zweiter Ordnung zu beschränken, die in manchen Problemen (als Beispiel sei die Kleinwinkelstreuung durch Schraubenversetzungen genannt) jedoch den Hauptbeitrag gibt. Auch hier kann man durch geeignete Umformungen die für die direkte Anwendung von Gl. (1) erforderliche, aber oft sehr mühselige Ermittlung von $\Theta(\mathfrak{r})$ umgehen, obschon die endgültige Formel weniger einfach als diejenige der „linearen“ Theorie (Gl. (17), s. u.) ist. Wir werden darauf an anderer Stelle eingehen, da in der vorliegenden Arbeit die Besprechung der nichtlinearen Elastizitätstheorie zuviel Raum beanspruchen würde.

Wir formen Gl. (1) mit Hilfe des Greenschen

\footnotetext{
6 A. SEeger, Z. Naturforschg. 11 a, 724 [1956].

7 A. Seeger, Acta Met. 5, 24 [1957].

8 J. Blin, Acta Met. 5, 528 [1957].

9 H. H. Atrinson u. P. B. Hirsch, Phil. Mag. 3, 213 [1958].

10 H. H. Atrinson u. P. B. Hirsch, Phil. Mag. 3, 862 [1958].
} 


\section{Satzes}

$$
\begin{aligned}
\iiint \psi \Delta \varphi \mathrm{d} \tau_{\mathrm{r}} & =\iiint \varphi \Delta \psi \mathrm{d} \tau_{\mathrm{r}} \\
& +\iint_{0}(\psi \operatorname{grad} \varphi-\varphi \operatorname{grad} \psi) \cdot \mathrm{d} \tilde{\mathfrak{T}}
\end{aligned}
$$

um, wobei wir die Dilatation $\Theta(r)$ mit $\psi$ identifizieren und

$$
\text { also } \varphi=-\frac{\exp (-i \mathfrak{g} \cdot \mathfrak{r})}{g^{2}}
$$

setzen. Das über die Oberfläche $O$ des betrachteten Körpers mit nach außen weisendem Normalvektor $\mathrm{d} \tilde{j}$ zu erstreckende Integral auf der rechten Seite von Gl. (4) ist dann Null, wenn wir einen nach allen Seiten unendlich ausgedehnten Körper betrachten, in dessen Innern eine endliche $Z$ ahl von geschlossenen Versetzungsringen liegt. Dann fallen nämlich die Verzerrungen nach außen mindestens wie $1 / r^{3}$ und deren Gradient mindestens wie $1 / r^{4}$ ab. Unter diesen Voraussetzungen geht also Gl. (1) über in

$$
A(\mathfrak{g})=-\iiint \Delta \Theta(\mathrm{r}) \frac{\exp (-i \mathfrak{g} \cdot \mathrm{r})}{g^{2}} \mathrm{~d} \tau_{\mathrm{r}} .
$$

Von jetzt ab werden wir die - für die Ableitung von Gl. (6) noch nicht nötige - Voraussetzung machen, daß sich $\Theta(\mathfrak{r})$ aus der linearen Elastizitätstheorie ergibt.

Wir betrachten ein homogenes, isotropes Medium des Schubmoduls $G$ und der Poisson-Zahl $v$. Bekanntlich erfüllt in einem solchen Medium die Volumendilatation $\Theta$ die Laplacesche Gleichung $\Delta \Theta=0$ in denjenigen Gebieten, in denen keine Volumenkräfte angreifen und die Kompatibilitätsbedingungen von DE ST. Venant nicht verletzt sind. Volumenkräfte werden in dieser Arbeit nicht betrachtet. Die Anwesenheit von Versetzungen hat jedoch im allgemeinen eine Verletzung der Kompatibilitätsbedingungen zur Folge, an deren Stelle die allgemeineren Gleichungen ${ }^{3}$

$$
\text { Ink } \boldsymbol{\epsilon} \equiv \nabla \times \boldsymbol{\epsilon} \times \nabla=\boldsymbol{\eta}
$$

treten, welche besagen, daß jetzt die Inkompatibilität des Deformationsfeldes $\boldsymbol{\epsilon}$ nicht mehr Null, sondern gleich einem symmetrischen Tensor 2. Stufe $\eta$ ist. $\eta$ bestimmt sich aus einer gegebenen Versetzungsdichte $\boldsymbol{\alpha}$ gemäß3

$$
\eta=\operatorname{Sym}(\boldsymbol{\alpha} \times \nabla) \text {. }
$$

Wir haben jetzt die Beziehung aufzusuchen, die bei nichtverschwindendem $\eta$ an die Stelle von
$\Delta \Theta=0$ tritt. Dazu schreibt man die Kompatibilitätsgleichungen in der Beltramischen Form. Bei $\eta \neq 0$ gilt $^{11} \quad(\boldsymbol{\sigma}=$ Spannungstensor $)$

$$
\Delta \boldsymbol{\sigma}+\frac{1}{1+v}\left(\nabla \nabla \sigma_{\mathrm{I}}-\Delta \sigma_{\mathrm{I}} \boldsymbol{I}\right)=2 G \boldsymbol{\eta} .
$$

I ist der Einheitstensor 2. Stufe; der Index I deutet immer den ersten Skalar des betreffenden Tensors an.

Bildet man den 1. Skalar von Gl. (9), so wird

$$
\frac{1-r}{1+r} \Delta \sigma_{\mathrm{I}}=-2 G \eta_{\mathrm{I}}
$$

und mit

$$
\Theta \equiv \varepsilon_{\mathrm{I}}=\begin{array}{cr}
1 & 1-2 v \\
2 G & 1+v
\end{array} \sigma_{\mathrm{I}}
$$

erhält man

$$
\Delta \Theta=-\frac{1-2 v}{1-v} \eta_{\mathrm{I}}=-\frac{1-2 v}{1-v}(\boldsymbol{\alpha} \times \nabla)_{\mathrm{I}} .
$$

Dies in Gl. (6) eingesetzt ergibt

$A(\mathfrak{g})=+\frac{1-2 v}{1-v} \iiint(\boldsymbol{\alpha} \times \nabla)_{\mathrm{I}} \frac{\exp (-i \mathfrak{g} \cdot \mathrm{r})}{g^{2}} \mathrm{~d} \tau_{\mathrm{r}}$

und mit partieller Integration, da das Oberflächenintegral wieder verschwindet,

$$
A(\mathfrak{g})=i \frac{1-2 v}{1-v} \iiint(\boldsymbol{\alpha} \times \mathfrak{g})_{\mathrm{I}} \frac{\exp (-i \mathfrak{g} \cdot \mathfrak{r})}{g^{2}} \mathrm{~d} \tau_{\mathrm{r}} .
$$

Diese Formel gestattet, die Kleinwinkelstreuung für räumliche oder flächenhafte Versetzungsanordnungen zu berechnen. Für eine singuläre Versetzung vom Burgers-Vektor $\mathfrak{b}$ (bei der also $\boldsymbol{\alpha}$ nur längs einer Linie von Null verschieden ist), wird

$$
\boldsymbol{\alpha}=\mathrm{t} \delta(p) \delta(q) \mathfrak{b},
$$

wo $p$ und $q$ geeignet gewählte Koordinaten senkrecht zum Linienverlauf $t$ der Versetzungslinie sind. Ist $\mathrm{d} l=\mathrm{t} \mathrm{d} l$ das vektorielle Linienelement der Versetzung, so hat man $\mathrm{t} d \tau_{\mathrm{r}}=\mathrm{d} l \mathrm{~d} p \mathrm{~d} q$, und

$$
\begin{array}{r}
A(\mathfrak{g})=i \frac{1-2 v}{1-v} \iiint(\mathrm{d} l \mathfrak{b} \times \mathfrak{g})_{\mathrm{I}} \delta(p) \delta(q) \\
\cdot \frac{\exp (-i \mathfrak{g} \cdot \mathfrak{r})}{g^{2}} \mathrm{~d} p \mathrm{~d} q .
\end{array}
$$

Die Integrationen über $p$ und $q$ ergeben sich von selbst, man erhält schließlich

$$
A(\mathfrak{g})=i \frac{1-2 v}{1-v} \frac{(\mathfrak{b} \times \mathfrak{g})}{g^{2}} \cdot \int \exp (-i \mathfrak{g} \cdot \mathfrak{l}) \mathrm{d} \mathfrak{l} .
$$

11 E. KRöNER ${ }^{3}$, Gl. (II. 22). 
Gl. (17) gibt die Amplitude der Kleinwinkelstreuung als Linienintegral über die Versetzung an. Dabei tritt das Spatprodukt $(\mathfrak{b} \times \mathfrak{g}) \cdot d \mathfrak{l}$ aus BurgersVektor $\mathfrak{b}$, Beugungsvektor $\mathfrak{g}$ und vektoriellem Linienelement dl der Versetzungslinie auf. Aus den Eigenschaften des Spatprodukts erhält man neben dem trivialen Resultat, das in einem isotropen Medium in erster Näherung eine Schraubenversetzung ( $\mathfrak{b}$ parallel zu dl) keinen Beitrag zur Kleinwinkelstreuung gibt, das Ergebnis, daß die Kleinwinkelstreuung dann Null ist, wenn der Beugungsvektor $\mathfrak{g}$ in der durch Burgers-Vektor und Linienelement aufgespannten Ebene, also der Gleitebene der Versetzung, liegt.

\section{Kleinwinkelstreuung durch einen kreis- förmigen Stufenversetzungsring}

Aus Gl. (17) kann man eine ganze Reihe in der Literatur bekannter Ergebnisse über die Kleinwinkelstreuung von Versetzungslinien sehr leicht ableiten. Wir wollen hier nur ein praktisch wichtiges, aber wegen zu großer mathematischer Schwierigkeiten bisher ungelöstes Problem mit Hilfe von Gl. (17) behandeln, nämlich die Kleinwinkelstreuung durch einen kreisförmigen Versetzungsring mit Radius $\varrho_{0}$ in einem isotropen Medium, wobei der BurgersVektor senkrecht auf der Kreisebene stehen soll. Ein solcher Stufenversetzungsring entsteht, wenn man eine kreisförmige Platte von Zwischengitteratomen in einen Kristall einbaut bzw. eine entsprechende Schicht von Atomen (etwa durch Kondensation von Leerstellen) entfernt.

Macht man die Richtung der Projektion von $\mathfrak{g}$ auf die Kreisringebene zur Achse eines ebenen $\varrho, \varphi$-Polarkoordinatensystems und bezeichnet den Betrag jener Projektion mit $g_{\rho}$, so nimmt im vorliegenden Falle Gl. (17) die Form

$$
\begin{aligned}
& A(\mathfrak{g})= \\
& \frac{1-2 v}{1-v} \\
& \frac{i b g_{\varrho} \varrho_{0}}{g^{2}} \int_{0}^{2 \pi} \exp \left(-i g_{\varrho} \varrho_{0} \cos \varphi\right) \cos \varphi \mathrm{d} \varphi,
\end{aligned}
$$

an. Diese läßt sich mit Hilfe der Bessel-Funktion 1. Ordnung $J_{1}(z)$ als

$$
A(\mathfrak{g})=\begin{array}{cc}
1-2 v & 2 \pi b g_{\varrho} \varrho_{0} \\
1-v & g^{2}
\end{array} J_{1}\left(g_{\varrho} \varrho_{0}\right)
$$

schreiben. Aus Gl. (19) folgt

$$
A(0)=\frac{1-2 v^{\prime}}{1-v} \pi b Q_{0}{ }^{2}\left(g_{\varrho} / g\right)^{2} .
$$

Zum Vergleich mit Beobachtungen hat man die zu $|A|^{2}$ proportionale Intensität der Streuung zu betrachten. Außerdem muß man natürlich in Rechnung stellen, daß in Wirklichkeit wohl immer Versetzungsringe verschiedener Orientierung vorliegen. Entstehen die Ringe beispielsweise durch die Kondensation von Leerstellen in einem kubisch-flächenzentrierten Kristall, so dürften sie in gleicher Weise auf die vier $\{111\}$-Ebenen verteilt sein. Wir wollen der Einfachheit halber annehmen, daß alle Orientierungen der Ringe gleich häufig vorkommen, was bedeutet, daß wir über alle Richtungen von $\mathfrak{g} z u$ mitteln haben. Die Intensität hängt dann nur noch vom Betrag von $\mathfrak{g}$ ab und ist proportional $\mathrm{zu}$

$$
I(g)=\frac{1}{4 \pi} \iint|A(\mathfrak{g})|^{2} \mathrm{~d} O_{\mathrm{g}},
$$

wobei das Integral über die Oberfläche der Einheitskugel im g-Raum zu erstrecken ist. Führt man sphärische Polarkoordinaten im g-Raum ein, so kann man Gl. (21) umformen in

$$
\begin{aligned}
I(g)=\left(\frac{1-2 v}{1-v}\right)^{2} 4 \pi^{2}\left(\frac{b \varrho_{0}}{g}\right)^{2} \int_{0}^{\pi / 2} \sin ^{3} \vartheta \\
\\
\cdot J_{1}^{2}\left(g \varrho_{0} \sin \vartheta\right) \mathrm{d} \vartheta .
\end{aligned}
$$

Man erkennt, daß $I(g)$ im Endlichen nicht Null wird, aber (da der Hauptbeitrag zum Integral in Gl. (22) aus der Umgebung von $\vartheta=\pi / 2$ kommt) in der Umgebung der Nullstellen von $J_{1}\left(g \varrho_{0}\right)$ (mit Ausnahme von $g=0$ ) sehr kleine Werte annimmt. Die ersten beiden dieser Nullstellen liegen bei $g \varrho_{0}=3,832$ und $g \varrho_{0}=7,016$.

Aus der Gleichung

$$
\begin{aligned}
& \frac{\mathrm{d} I(g)}{\mathrm{d} g}=-\left(\frac{1-2 v}{1-v}\right)^{2}\left(\begin{array}{c}
b \varrho_{0} \\
g
\end{array}\right)^{2} 8 \pi^{2} \varrho_{0} \\
& \quad \cdot \int_{0}^{\pi / 2} \sin ^{4} \vartheta J_{1}\left(g \varrho_{0} \sin \vartheta\right) J_{2}\left(g \varrho_{0} \sin \vartheta\right) \mathrm{d} \vartheta
\end{aligned}
$$

entnimmt man, daß Maxima von $I(g)$ in der Nähe der Nullstellen von $J_{2}\left(g \varrho_{0}\right)$ auftreten, deren erste drei bei $g \varrho_{0}=0, g \varrho_{0}=5,135$ und $g \varrho_{0}=8,417$ liegen. Für die auf $g=0$ extrapolierte Intensität ergibt sich

$$
I(0)=\left(\frac{1-2 v}{1-v}\right)^{2} \frac{8 \pi^{2}}{15} b^{2} \varrho_{0}{ }^{4} .
$$

Wendet man die Formel ${ }^{12}$

12 W. N. Barley, Quart. J. Math., Oxford Ser. 9, 141 [1938]. 
$z \int_{0}^{\pi / 2} J_{\nu}^{2}(z \sin \vartheta) \sin ^{2 v+1} \vartheta \mathrm{d} \vartheta=\frac{\Gamma(2 v+1)}{2^{2} v+1\{\Gamma(v+1)\}^{2}} \sum_{n=0}^{\infty} \frac{\Gamma\left(n+\frac{1}{2}\right) \Gamma(2 v+n+1)}{n ! \Gamma\left(2 v+n+\frac{3}{2}\right)}(2 v+2 n+1) J_{2 v+2 n+1}(2 z)$

an, so erhält man $I(g)$ als Neunannsche Reihe in der Form

$$
I(g)=\left(\frac{1-2 v}{1-v}\right)^{2} 8 \pi^{2} \frac{b^{2} \varrho_{0}}{g^{3}} \sum_{n=0}^{\infty} \frac{(n+2)(n+1)}{(2 n+5)(2 n+1)} J_{2 n+3}\left(2 g \varrho_{0}\right) .
$$

Gl. (26) eignet sich gut zur Berücksichtigung einer Verteilung der auftretenden Ringradien $\varrho_{0}$ und zur numerischen Auswertung für nicht zu große $g \varrho_{0}$. Der Grenzwert der Summe in Gl. (26) für $g \varrho_{0} \rightarrow \infty$ ist $1 / 8$, so daß $I$ ( $g$ ) für große $g$ wie $g^{-3}$ abnimmt.

Den eben erwähnten Grenzwert sowie weitere Glieder der asymptotischen Entwicklung von $I(g)$ kann man auf folgende Weise ableiten:

Aus der bekannten Formel

$$
\int_{0}^{x} J_{v}(x) \mathrm{d} x=2 \sum_{n=0}^{\infty} J_{v+2} n+1(x)
$$

erhält man

$$
-\frac{1}{4} \sum_{n=0}^{\infty} J_{\nu+2} n+1(z)+\frac{1}{8}-\frac{1}{8} \int_{z}^{\infty} J_{v}(x) \mathrm{d} x=0 .
$$

Kombiniert man Gl. (28) mit Gl. (26), so ergibt sich

$$
\begin{aligned}
I(g)= & \left(\frac{1-2 v}{1-v}\right)^{2} \frac{\pi^{2} b^{2} \varrho_{0}}{g^{3}} \\
& \cdot\left\{1-\int_{2 g \varrho_{0}}^{\infty} J_{2}(x) \mathrm{d} x+6 \sum_{n=0}^{\infty} \frac{J_{2 n+3}\left(2 g \varrho_{0}\right)}{4 n^{2}+12 n+5}\right\} .
\end{aligned}
$$

Durch wiederholte partielle Integration des in Gl. (29) auftretenden Integrals sowie durch Einsetzen der Integraldarstellung der Besset-Funktionen in die unendliche Reihe, Vertauschen von Summation und Integration und Anwendung der Sattelpunktsmethode ergibt sich eine asymptotische Entwicklung nach fallenden Potenzen von $g$, die, abgesehen von dem Faktor $g^{-3}$, dieselbe allgemeine Form wie diejenige der Zylinderfunktionen hat.

\section{Diskussion}

Das Hauptergebnis der vorliegenden Arbeit ist die Ableitung der Gl. (17), die im Rahmen der linearen Elastizitätstheorie für elastisch isotrope Medien gilt. Die Erweiterung auf Kristalle kubischer Symmetrie wird in einem Anhang gegeben. Die Darstellung der von einer Versetzungsanordnung hervorgerufenen Kleinwinkelstreuung durch ein Linienintegral bietet neben der Vereinfachung der Rechnungen bei speziellen Problemen offenkundige Vorteile für die physikalische Diskussion: Man kann auf diese Weise verhältnismäßig einfach die Interferenz zwischen benachbarten Versetzungslinien, etwa in aufgespaltenen Versetzungen, in Kleinwinkelkorngrenzen oder in aufgestauten Versetzungsgruppen, behandeln. Sind die Versetzungen so nahe benachbart, daß man die Individualität der einzelnen Versetzungen nicht zu berücksichtigen braucht, so empfiehlt sich die Anwendung der für flächenhafte oder räumliche kontinuierliche Versetzungsverteilungen geltenden Gl. (14) .

Die Gln. (14) und (17) lassen sich auch auf andere Gebiete anwenden, z. B. den elektrischen Widerstand von Versetzungen in Metallen. Beschreibt man die Leitungselektronen in einem Metall als ein Gas freier Elektronen, so ist das von einer Versetzung hervorgerufene Streupotential proportional zur Volumdilatation. In der Bonsschen Näherung wird somit die Streuwahrscheinlichkeit eines „freien“ Elektrons, dessen Ausbreitungsvektor sich bei der Streuung um $\mathfrak{g}$ ändert, proportional zu $A(\mathfrak{g})$. Man kann den Ergebnissen der vorliegenden Arbeit entnehmen, daß in dieser Näherung die Streuwahrscheinlichkeit Null ist, wenn $\mathfrak{g}$ in der Gleitebene der Versetzung liegt oder senkrecht auf der Ebene einer ringförmigen Stufenversetzung steht.

Bei dem in Abschnitt 2 behandelten speziellen Beispiel liegt ein Vergleich mit der Kleinwinkelstreuung durch kugelförmige Hohlräume nahe. Wie bereits erwähnt, können sich Stufenversetzungsringe (ebenso wie kugelförmige Hohlräume) durch Kondensation von Leerstellen bilden, so daß eine Untersuchung des Unterschieds in der Kleinwinkelstreuung durch die genannten beiden Fehlstellen erwünscht erscheint.

Ein Stufenversetzungsring mit Radius $\varrho_{0}$ und Burgers-Vektor $\mathfrak{b}$ entsteht in einem Kristall mit dem Atomvolumen $\Omega$ durch Kondensation von $Z=\pi b \varrho_{0}^{2} / \Omega$ Leerstellen. Eine Kugel, die die gleiche 
Anzahl von leeren Gitterplätzen enthält, hat einen Radius $R=\left(\frac{3 b \varrho_{0}^{2}}{4}\right)^{1 / 3}$. Das Verhältnis der auf den Streuwinkel Null extrapolierten Intensitäten von Versetzungsring und Kugel ist

$$
\omega \equiv I_{\text {Vers. }}(0) / I_{\text {Kugel }}(0)=\frac{8}{15}\left(\frac{1-2 v}{1-v}\right)^{2},
$$

beträgt also für ein Medium mit $v=1 / 3, \omega=0,133$. Die Ringe streuen also bei kleinen Streuwinkeln erheblich weniger als Kugeln, die aus derselben Anzahl von Leerstellen entstanden sind ${ }^{13}$. Für große Streuwinkel nimmt die Streuintensität der Kugeln wie $g^{-4}$, diejenige der Ringe wie $g^{-3} \mathrm{ab}^{\mathbf{1 4}}$; das asymptotische Verhältnis der Streuintensitäten von Ringen und Kugeln mit gleichem $Z \Omega$ beträgt $\left(\frac{1-2 v}{1-v}\right)^{2} \frac{b g}{6}\left(\frac{3 b}{4 \varrho_{0}}\right)^{1 / 3}$. Dieses Verhältnis wird größer als Eins erst bei Streuwinkeln $2 \vartheta$, bei denen man die Kleinwinkelstreuung nicht mehr messen kann. Immerhin sollte das verschiedenartige asymptotische Verhalten von $I(g)$ die experimentelle Entscheidung darüber erleichtern, ob bei einer bestimmten Untersuchung Versetzungsringe oder Hohlräume vorliegen.

Die vorstehenden Ausführungen haben durch kürzliche Beobachtungen von Hirsch, Silcox, Smallman u. Westmacotr ${ }^{15}$, die in dünnen, von hohen Temperaturen abgeschreckten Aluminiumfolien elektronenmikroskopisch kleine Versetzungsringe sichtbar machen konnten, sehr an Interesse gewonnen. SmaLLMAN konnte ferner zeigen, daß solche Proben, die bei Raumtemperatur angelassen worden waren, im Bereich von $\vartheta=1^{\circ} / 4$ eine Kleinwinkelstreuung von RöNTGEN-Strahlen zeigten, die vielleicht von solchen Ringen herrührt. Nur Ringe mit einem Radius von weniger als etwa $100 \AA$ Radius geben in diesem Winkelbereich einen Beitrag zur Kleinwinkelstreuung, der vor dem ersten im Anschluß an Gl. (22) diskutierten Minimum $\left(g \varrho_{0}=3,8\right)$ liegt. Die mei-

13 Schon Dexter ${ }^{4}$ hat darauf hingewiesen, daß Stufenversetzungsringe, wie sie durch Kondensation von Leerstellen erzeugt werden können, zu einer endlichen Kleinwinkelstreuung bei kleinen Streuwinkeln Anlaß geben, da ja bei ihrer Bildung Materie entfernt und an die Kristalloberfläche gebracht wurde. $\mathrm{Da}$ die über alle Richtungen im g-Raum gemittelte Amplitude $A(0)$ (Gl. 20) nicht $Z \Omega=\pi b \varrho_{0}{ }^{2}$ ergibt, sondern noch mit dem Faktor $\frac{2}{3}\left(\frac{1-2 v}{1-v}\right)$ behaftet ist, hängt mit den elastischen Verzerrungen zusammen, die einen wesentlichen Teil der von den Leerstellen hervorgerufenen Volumänderungen rückgängig sten in den elektronenmikroskopischen Bildern zu sehenden Ringe sind größer, so daß die von diesen herrührende Kleinwinkelstreuung im Gültigkeitsbereich des $g^{-3}$-Gesetzes liegen dürfte. Legt man eine Konzentration von $10^{-5}$ eingefrorenen und $\mathrm{zu}$ Ringen kondensierten Leerstellen zugrunde, so erhält man in dem fraglichen Winkelbereich mit $\mathrm{CuK} \alpha$-Strahlung eine Intensität von etwa 1/10 Elektron/Atom, die wohl zu klein ist, um nachgewiesen werden zu können. Hirsch et al. vertreten deshalb die Auffassung, daß die beobachtete Kleinwinkelstreuung von doppelten BRAGG-Reflexionen herrührt. Es erscheint jedoch auch denkbar, daß sehr kleine kugelförmige Hohlräume, die im elektronenmikroskopischen Bild nicht sichtbar sind, zur Kleinwinkelstreuung beitragen.

\section{A n h a ng}

\section{Kleinwinkelstreuung durch Versetzungen in anisotropen Medien}

Bei Anisotropie der elastischen Konstanten hat man die Methode der räumlichen Spannungsfunktionen zu verwenden. Im Fall des kubischen Mediums, auf das wir uns beschränken, gilt wie bei Isotropie $\left(K=\frac{c_{11}+2 c_{12}}{3}=\right.$ Kompressionsmodul; $\quad c_{i k}=$ Vorgrsche elastische Konstanten)

$$
\Theta=\frac{1}{3 K} \sigma_{\mathrm{I}}
$$

Mit dem Ansatz $\sigma=\operatorname{Ink} \chi$ werden bekanntlich die Gleichgewichtsbedingungen identisch befriedigt. Dann ist

$$
\sigma_{\mathrm{I}}=(\nabla \nabla-\Delta \boldsymbol{I}) \cdots \chi
$$

Man setzt bei elastischer Anisotropie weiter ${ }^{16}$

$$
x=\mathbf{x} \cdot \boldsymbol{\psi}
$$

$\psi$ heißt Spannungsfunktionstensor 4. Ordnung. $\mathbf{X}(\nabla)$ ist ein tensorieller Differentialoperator 4. Stufe und 2. Ordnung von der gleichen Symmetrie wie der Hookesche Elastizitätstensor des betreffenden Mediums.

machen. Vgl. den analogen Fall einer Hohlkugel, deren Volumen durch die Oberflächenspannung verkleinert wird. 14 Das asymptotische Verhalten der von Versetzungsringen herrührenden Kleinwinkelstreuung liegt demnach zwischen demjenigen der Streuung von Kugeln $\left[I(g) \sim g^{-4}\right]$ und demjenigen der Streuung durch sehr dünne Scheiben $\left[g^{-1}\right.$ groß gegen Scheibendicke, $\left.I(g) \sim g^{-2}\right]$, siehe Guinier und Fournet ${ }^{1}$.

15 P. B. Hirsch, J. Silcox, R. E. Smallman u. K. H. Westmacott, Phil. Mag. 3, 897 [1958]. Wir danken Herrn Dr. R. Smallman für die freundliche Mitteilung der Beobachtungen vor der Veröffentlichung.

16 E. Kröner, Z. Phys. 141, 386 [1955]. 
Es ist im kubischen Fall

$$
\begin{aligned}
X_{x x x x} & =\left(a_{1}+a_{2}\right) \Delta+a_{3} \frac{\partial^{2}}{\partial x^{2}}, \quad X_{x x y y}=a_{2} \Delta, \\
2 X_{x y x y} & =a_{4} A+a_{5} \frac{\partial^{2}}{\partial z^{2}},
\end{aligned}
$$

usw. mit zyklischer Vertauschung von $x, y, z$. Dabei ist

$a_{1} \equiv c_{11} c_{44}^{2}\left(2 c_{44}+\mu^{\prime}\right)$,

$a_{2} \equiv c_{12} c_{44}{ }^{2}\left(2 c_{44}+\mu^{\prime}\right)$,

$a_{3} \equiv \mu^{\prime} c_{44}\left(c_{11}^{2}+c_{11} c_{12}-2 c_{12}^{2}-2 c_{12} c_{44}\right)$,

$a_{i} \equiv 2 c_{11} c_{44}{ }^{3}+\mu^{\prime} c_{44}{ }^{2}\left(c_{11}+c_{12}\right)$,

$$
\begin{aligned}
& a_{5} \equiv-\mu^{\prime} c_{44}{ }^{2}\left(c_{11}+c_{12}\right), \\
& \mu^{\prime} \equiv c_{11}-c_{12}-2 c_{44} .
\end{aligned}
$$

Mit (A 2) und (A 3) geht Gl. (A 1) in

$$
\begin{gathered}
\Theta=\boldsymbol{H}(\nabla) \cdots \boldsymbol{\psi} ; \\
\boldsymbol{H}(\nabla)=\frac{1}{3 K}(\nabla \nabla-\Delta \boldsymbol{I}) \cdots \mathbf{X}(\nabla)
\end{gathered}
$$

über. Nun befriedigt der Spannungsfunktionstensor $\psi$ die Differentialgleichung

$$
f(\nabla) \psi=\eta
$$

mit

$$
f(\nabla) \equiv c_{11} c_{44^{2}}{ }^{2}\left\{\Delta \Delta \Delta+\frac{\mu^{\prime}\left(c_{11}+c_{12}\right)}{c_{11} c_{44}}\left(\frac{\partial^{4}}{\partial x^{2} \partial y^{2}}+\frac{\partial^{4}}{\partial y^{2} \partial z^{2}}+\frac{\partial^{4}}{\partial z^{2} \partial x^{2}}\right) \Delta+\mu^{\prime 2} \frac{c_{11}+2 c_{12}+c_{44}}{c_{11} c_{44}{ }^{2}} \partial x^{2} \partial y^{2} \partial z^{2}\right\} .
$$

Gl. (A 5) geht daher nach Anwendung von $f(\nabla)$ in

$$
f(\nabla) \Theta=\boldsymbol{H}(\nabla) \cdots \eta
$$

über, bzw., wenn man die Inkompatibilitäten nach Gl. (8) durch die Versetzungsdichten $\boldsymbol{\alpha}$ ersetzt, in

$$
\begin{array}{r}
f(\nabla) \Theta=\boldsymbol{H}(\nabla) \cdots(\boldsymbol{\alpha} \times \nabla)=-\boldsymbol{H}(\nabla) \cdots(\nabla \times \tilde{\boldsymbol{\alpha}}) ; \\
\boldsymbol{\alpha}_{i j}=\tilde{\boldsymbol{\alpha}}_{j i} .
\end{array}
$$

Wir schreiben nun die Amplitude $A(\mathfrak{g})$ von Gl. (1) in der Form

$$
A(\mathfrak{g})=-\iiint \Theta(\mathrm{r})\left\{f(\nabla) \frac{\exp (-i \mathfrak{g} \cdot \mathrm{r})}{f(\mathfrak{g})}\right\} \mathrm{d} \tau \mathrm{r} .
$$

Partielle Differentiation ergibt, da die Oberflächenintegrale verschwinden,

$A(\mathfrak{g})=-\iiint\{f(\nabla) \Theta(\mathrm{r})\} \frac{\exp (-i \mathfrak{g} \cdot \mathfrak{r})}{f(\mathfrak{g})} \mathrm{d} \tau \mathrm{r}$.

Hier können wir nun Gl. (A 8) einführen und bekommen

$A(\mathfrak{g})=\iiint\{\boldsymbol{H}(\nabla) \cdot(\nabla \times \tilde{\boldsymbol{\alpha}})\} \frac{\exp (-i \mathfrak{g} \cdot \mathfrak{r})}{f(\mathfrak{g})} \mathrm{d} \tau \mathrm{r} \cdot($

Nochmalige partielle Integration liefert die Verallgemeinerung der Gl. (14) für kubische Medien:
$A(\mathfrak{g})=+i \boldsymbol{H}(\mathfrak{g}) \cdots \iiint \mathfrak{g} \times \tilde{\boldsymbol{\alpha}} \frac{\exp (-i \mathfrak{g} \cdot \mathfrak{r})}{f(\mathfrak{g})} \mathrm{d} \tau \mathfrak{r} \cdot($

Für die singuläre Versetzung ersetzt man wieder $\boldsymbol{\alpha}$ nach Gl. (15) und erhält die Verallgemeinerung von Gl. (17)

$$
A(\mathfrak{g})=i\left\{\frac{\boldsymbol{H}(\mathfrak{g}) \cdot[\mathfrak{g} \times \mathfrak{b}]}{f(\mathfrak{g})}\right\} \cdot \int \exp (-i \mathfrak{g} \cdot \mathrm{r}) \mathrm{d} \mathfrak{l} .
$$

Bemerkenswerterweise führt die Kleinwinkelstreuung durch Versetzungen im kubischen Medium auf das gleiche Linienintegral wie im isotropen Fall. Der Unterschied zu Gl. (17) besteht nur darin, daß, abgesehen von einer Konstanten, an die Stelle des Vektors $\mathfrak{a} \times \mathfrak{b}$ der modifizierte Vektor tritt, den man erhält, wenn man den Vektor $\mathfrak{g} \times \mathfrak{b}$ mit dem symmetrischen Tensor $\boldsymbol{H}(\mathfrak{g}) / f(\mathfrak{g})$ skalar multipliziert. Die cartesischen Komponenten von $H(\mathfrak{g})$ lauten

$$
\begin{aligned}
& H_{x x}(\mathfrak{g})=\frac{1}{3 K}\left\{-\left(a_{1}+2 a_{2}\right) g^{4}+\left(a_{1}-a_{3}\right) g^{2} g_{x}{ }^{2}+a_{3} g_{r^{4}}\right\} \\
& H_{x y}(\mathfrak{g})=\frac{1}{3 K} g_{x} g_{y}\left(a_{4} g^{2}+a_{5} g_{z}^{2}\right) .
\end{aligned}
$$

usw. mit zyklischer Vertauschung von $x, y, z$.

Der allgemeine anisotrope Fall wäre in gleicher Weise zu behandeln. Leider ist hier der Tensor $\mathbf{X}$ noch nicht berechnet worden, so daß man praktisch doch noch auf den kubischen Fall beschränkt bleibt. 